\title{
Editorial: Metallic Biomaterials for Medical Applications
}

\author{
Liqiang Wang ${ }^{1 *}$, Chaozong Liu $^{2}$ and Lechun Xie ${ }^{3,4}$ \\ ${ }^{1}$ State Key Laboratory of Metal Matrix Composites, School of Materials Science and Engineering, Shanghai Jiao Tong University, \\ Shanghai, China, ${ }^{2}$ Institute of Orthopaedic and Musculoskeletal Science, University College London, Royal National Orthopaedic \\ Hospital, Stanmore, United Kingdom, ${ }^{3}$ Hubei Key Laboratory of Advanced Technology for Automotive Components, Wuhan \\ University of Technology, Wuhan, China, ${ }^{4}$ Hubei Collaborative Innovation Center for Automotive Components Technology, \\ Wuhan, China
}

Keywords: biomaterials, medical applications, metal, titanium, magnesium

\section{Editorial on the Research Topic}

\section{Metallic Biomaterials for Medical Applications}

Biomaterials are natural or artificial materials used to make structures or implants to replace lost or diseased biological structures to restore form and function. In actual clinical situation, they are used in the complex environment of body fluid, so the requirements for the structure and properties of biomaterials are very strict. Biomaterials require great physical and chemical stability, however, some alloy elements, toxic and biological incompatible, harm the human body and some metals' mechanical properties don't match the human body. Therefore, for further improving the biological and mechanical properties of metallic biomaterials, many attempts have been adopted to design novel metallic biomaterials and different advanced processing and preparation technologies have been developed in the content of this topic.

Titanium alloys are widely used for dental and implant materials because of their superior biocompatibility, corrosion resistance and specific strength compared with other metallic implant materials. In the aspect of Ti alloys, Liu et al. investigated the antibacterial properties of various surface nano-modification of Ti implant materials and the related procedures, and the surface nanomodification of $\mathrm{Ti}$ materials were discussed from three aspects: nanostructures formation procedures, nanomaterials loading, and nano-morphology. Cheng et al. investigated a biomedical beta type $\mathrm{Ti}-25 \mathrm{Nb}-3 \mathrm{Zr}-2 \mathrm{Sn}-3 \mathrm{Mo}$ alloy plate via cold rolling deformation, and the alloy plates possessed various mechanical properties owing to the different morphology, size and volume fraction of a precipitated phases, which would be deemed as a potential material in the orthopedic field. Cheng et al. also investigated the microstructure evolution and mechanical properties of metastable beta type titanium alloy (Ti-B12) after different aging treatments. MTT test with L929 cells showed that the cytotoxicity of Ti-B12 was better than that of Ti-6Al-4V, which was suitable for biomedical application.

As lightweight alloys with mechanical properties close to human bone and degradable in the human environment, magnesium alloys have good biocompatibility and are widely used in load-bearing implants and degradable stents. In terms of $\mathrm{Mg}$ alloys, Ibrahim et al. introduced a $\mathrm{Mg} / \mathrm{SrP}$ composite coating on Ti-6Al-4V in order to promote the bone tissue healing and shorten the healing cycle; the results revealed that $\mathrm{Mg} / \mathrm{SrP}$ coating shows more suitable degradation rate than pure $\mathrm{Mg}$, with no cytotoxicity and higher proliferation compared with the culture medium. Qiao et al. fabricated $\mathrm{Mg} / \mathrm{ZrO}_{2}$ metal matrix nanocomposites by friction stir processing (FSP), and the $\mathrm{Mg} / \mathrm{ZrO}_{2}$ achieved homogenization, densification, and grain refinement after FSP, which are due to the grain refinement and Orowan strengthening. $\mathrm{Wu}$ et al. used calcium phosphates ( $\mathrm{CaPs}$ ) coated on the surface of pure Mg through a simple alkali-hydrothermal treatment, and the $\mathrm{CaP}$ coating of $\mathrm{Mg}$ favors cell attachment and cell 
spreading, which can endow Mg with higher surface energy and osteogenesis capability and lower degradation than pristine $\mathrm{Mg}$.

In order to meet the biological, chemical, and mechanical properties of actual clinical needs, it is necessary to perform surface modification treatment on the above-mentioned metallic biomaterials. Surface treatment methods are very important methods to modify the physical and mechanical properties of biomaterials. Xue et al. reviewed the main physical and chemical surface modification techniques for Ti related biomaterials, such as plasma spray, PIII, PIII\&D, PVD, CVD, sol-gel and MAO, and future studies were introduced and should be focused on designing the basic new methods or the combination of modification methods to play a synergistic effect and combine their advantages to conquer the deficiencies. Delannoy et al. developed a thermomechanical strategy to create a radial elasticity gradient in a $\beta$ metastable $\mathrm{Ti}-\mathrm{Nb}-\mathrm{Zr}$ alloy, and shot-peening enabled to locally induce martensitic transformation on surface, and a decrease in indentation elastic modulus; this combination of material and process could be suitable to produce dental implants with mechanically enhanced biocompatibility. Yi et al. studied the characteristics of passive films formed on as-cast Ti-6Al-4V before and after transpassivation by electrochemical methods, which advance the understanding of as-cast Ti-6Al-4V polarized under different potentials for potential biomedical applications.

In addition to mainstream titanium alloys and magnesium alloys, efforts should be made to develop novel metallic biomaterials. In the aspects of new alloys and manufacturing methods, Ma et al. provided an insight into the development of Ti-based high entropy alloy (HEA), and the emergence of HEAs has brought great room for development in the field of medical implant materials. Shi et al. reviewed the current scenery of functional gradient metallic materials (FGM) in the biomedical field, specifically its dental and orthopedic applications; any improvement in FGM can lead to big steps toward its industrialization and most notably for much better implant designs with more biocompatibility and similarity to natural tissues. Xu et al. investigated the copper-containing alloy as immunoregulatory material in bone regeneration via mitochondrial oxidative stress, and the copper-containing metal promoted the evolution of callus through the generation of type $H$ vessels during the process of bone repair by upregulating the expression of PDGF$\mathrm{BB}$ derived from M2a macrophages. Attarilar et al. reviewed the related factors in toxicity of nanoparticles such as size, concentration, etc. with an emphasis on metal and metal oxide nanoparticles, which can highlight their potential hazard and the advancement of green non-cytotoxic nanomaterials with safe threshold dose levels to resolve the toxicity issues.
With the maturity of additive manufacturing technology, we should focus on developing complex, gradient and multiscale structures to customize medical devices. Some special structures were utilized to realize the medical application. Lv et al. reviewed the research progress of porous metal scaffolds, and introduced the additive manufacturing technology used in porous metal scaffolds, which provided unprecedented opportunities for production of customized biomedical implants. Zou et al. studied a novel design of temporomandibular joint (TMJ), and an artificial TMJ prosthesis designed with a porous structure on the condylar neck region for lateral pterygoid muscle (LPM) attachment was fabricated by a $3 \mathrm{D}$ printed $\mathrm{Ti}$ alloy; The TMJ prosthesis can help LPM attach to its porous titanium scaffold structure area for future function.

The contributions of above work were summarized as following: the important metallic biomaterials, especially $\mathrm{Ti}$ and $\mathrm{Mg}$ alloys; the surface treatments on biomaterials; some new alloys and manufacturing methods; and some special structures for special application. This research topic focused on the recent development of metallic biomaterials for medical applications, and all results would promote the development of metallic biomaterials.

\section{DATA AVAILABILITY STATEMENT}

The original contributions presented in the study are publicly available in NCBI under accession number PRJNA591354.

\section{AUTHOR CONTRIBUTIONS}

LW, CL, and LX wrote the original manuscript and revised the manuscript. They contribute equally.

Conflict of Interest: The authors declare that the research was conducted in the absence of any commercial or financial relationships that could be construed as a potential conflict of interest.

Publisher's Note: All claims expressed in this article are solely those of the authors and do not necessarily represent those of their affiliated organizations, or those of the publisher, the editors and the reviewers. Any product that may be evaluated in this article, or claim that may be made by its manufacturer, is not guaranteed or endorsed by the publisher.

Copyright (c) 2021 Wang, Liu and Xie. This is an open-access article distributed under the terms of the Creative Commons Attribution License (CC BY). The use, distribution or reproduction in other forums is permitted, provided the original author(s) and the copyright owner(s) are credited and that the original publication in this journal is cited, in accordance with accepted academic practice. No use, distribution or reproduction is permitted which does not comply with these terms. 